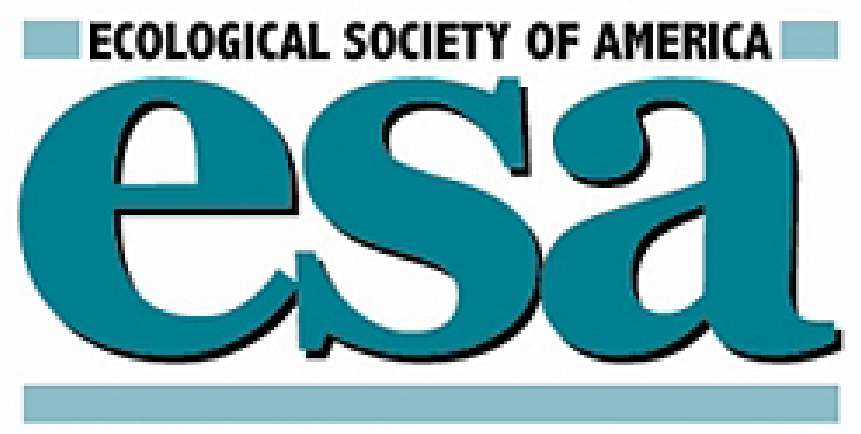

The Role of the Delay Time in the Modeling of Biological Range Expansions Author(s): Vicente Ortega-Cejas, Joaquim Fort and Vicenç Méndez

Reviewed work(s):

Source: Ecology, Vol. 85, No. 1 (Jan., 2004), pp. 258-264

Published by: Ecological Society of America

Stable URL: http://www.jstor.org/stable/3450486

Accessed: 06/02/2013 07:37

Your use of the JSTOR archive indicates your acceptance of the Terms \& Conditions of Use, available at http://www.jstor.org/page/info/about/policies/terms.jsp

JSTOR is a not-for-profit service that helps scholars, researchers, and students discover, use, and build upon a wide range of content in a trusted digital archive. We use information technology and tools to increase productivity and facilitate new forms of scholarship. For more information about JSTOR, please contact support@ jstor.org. 


\title{
THE ROLE OF THE DELAY TIME IN THE MODELING OF BIOLOGICAL RANGE EXPANSIONS
}

\author{
Vicente Ortega-Cejas, ${ }^{1,4}$ Joaquim Fort, ${ }^{2}$ and Vicenç Méndez ${ }^{3}$ \\ ${ }^{1}$ Grup de Física Estadística, Departamento de Física, Universitat Autònoma de Barcelona, 08193 Bellaterra, Spain \\ ${ }^{2}$ Departamento de Física, Universitat de Girona, 17071 Girona, Catalonia, Spain \\ ${ }^{3}$ Facultat de Ciències de la Salut, Universitat Internacional de Catalunya, Gomera s/n, 08190 Sant Cugat del Vallès, \\ Catalonia, Spain
}

\begin{abstract}
The time interval between successive migrations of biological species causes a delay time in the reaction-diffusion equations describing their space-time dynamics. This lowers the predicted speed of the waves of advance, as compared to classical models. It has been shown that this delay-time effect improves the modeling of human range expansions. Here, we demonstrate that it can also be important for other species. We present two new examples where the predictions of the time-delayed and the classical (Fisher) approaches are compared to experimental data. No free or adjustable parameters are used. We show that the importance of the delay effect depends on the dimensionless product of the initial growth rate and the delay time. We argue that the delay effect should be taken into account in the modeling of range expansions for biological species.
\end{abstract}

Key words: Carpodacus mexicanus; delay time; Eurasian Collared-Dove; House Finch; range expansions; Streptopelia decaocto; wavefront speed.

\section{INTRODUCTION}

The spread of biological species is a problem of utmost ecological importance. The classical mathematical formulation of these phenomena is due to Fisher (1937). Fisher's model has been applied to human range expansions (Ammerman and Cavalli-Sforza 1984, Rendine et al. 1986) and other species (Hengeveld 1989, Shigesada and Kawasaki 1997). On the other hand, individuals reproduce themselves, but it takes some time for the subsequent generation to migrate and reproduce. Fort and Méndez $(1999 a, b)$ showed that this implies that Fisher's model should be refined. The resulting time-delayed theory has up to now been compared to observations only for humans (Fort and Méndez 1999a, Fort 2003, Fort et al. 2003).

Time delays have been widely used in biology (e.g., in population dynamics) but seldom in the prediction of the speed of population expansions. Holmes (1993) modeled spatial spread using the telegrapher's equation, which she derived under the assumption of a correlated random walk. She found negligible changes $(<8 \%)$ to the classical predictions for five species (cabbage butterfly, Eurasian Collared-Dove, etc.). She did not analyze the delay due to the rest time between subsequent migrations (generations). Kot and coworkers (Kot 1992, Kot et al. 1996) have derived many interesting theoretical results (such as accelerating invasions and front shapes) using integrodifference equations, which, as discrete-time models, nat-

Manuscript received 1 October 2002; revised 16 April 2003; accepted 3 May 2003. Corresponding Editor: M. G. Neubert.

${ }^{4}$ E-mail: vicente.ortega@uab.es urally incorporate a delay. Neubert and Caswell (2000) developed a method to determine the speed of the wave front for a model resting on integrodifference equations for structured populations. Van den Bosch et al. (1990, 1992) developed continuous-time age-structured models and applied them to the expansion of populations and plant diseases. Thus, progress has been made on delay times and biological invasions. But the effect of the rest time between successive migrations (generations) has so far been taken into account in telegraph-dispersal models, only for human expansions.

Here, we will consider two avian range expansions, of Eurasian Collared-Dove Streptopelia decaocto and House Finch Carpodacus mexicanus, and compare their observed range expansion rates to the predictions of both Fisher's equation and the time-delayed telegraph dispersal model developed here. For these birds, reproduction does not occur throughout the year; indeed it is episodic and relatively synchronous. Therefore, one way to model their spread would be the building of a discrete-time model. Clearly, this would be necessary if the time scale of measurements were of the order of (or lower than) the typical reproduction time (i.e., about a year). However, measurements of population invasion speeds usually span several decades. Under those conditions it is a reasonable approximation of the use of a continuous model for bird reproductive dynamics.

\section{Time-Delayed EXTENSION OF FisheR's TheORY}

Traditionally Fick's law has been used to establish the relation between population flux, $\mathbf{J}$, and the population density gradient, $\Delta p$. This "law" hypothesizes 
a linear relationship between the two, via a factor known as the diffusion coefficient, $D$ :

$$
\mathbf{J}(\mathbf{x}, t)=-D \nabla p(\mathbf{x}, t) .
$$

In addition to motion, reproduction and mortality also contribute to changes in the density of individuals at a given point in space. In differential form, this corresponds to the well-known equation

$$
\frac{\partial p(\mathbf{x}, t)}{\partial t}=-\nabla \cdot \mathbf{J}(\mathbf{x}, t)+F[p(\mathbf{x}, t)]
$$

where $F[p]$ is the source term and accounts for the births and deaths per unit area. From Eqs. 1 and 2 one finds

$$
\frac{\partial p(\mathbf{x}, t)}{\partial t}=D \nabla^{2} p(\mathbf{x}, t)+F[p(\mathbf{x}, t)] .
$$

This is Fisher's equation. It is also known as the parabolic reaction-diffusion equation (PRDE).

Fick's law (Eq. 1) implies an instantaneous response to a suddenly established population gradient, thus neglecting the inertia and other possible additional sources of delay when dealing with biological individuals. On the other side, when the mean-free-path of the individuals (i.e., the average length of the path they may follow without change in direction) becomes indefinitely long (the so-called ballistic limit in physical literature), Fick's law predicts a divergent flux, because the diffusion coefficient diverges in this case (as it is proportional to the square of the mean-freepath). In contrast, the actual maximum possible value for the flux of individuals is the maximum individual speed times their number density. Both features (instantaneous response and divergent flux) may be avoided if delay effects are included (Jou et al. 2001). This delay takes into account the time that the individuals need in order to adopt a definite mean speed and direction of motion after nonuniformity appears in their concentration. Then Fick's law (Eq. 1) is replaced by

$$
\mathbf{J}(\mathbf{x}, t+\tau)=-D \Delta p(\mathbf{x}, t)
$$

where $\tau$ is the delay time. Again we can make use of the balance equation (Eq. 2), which now leads to

$$
\frac{\partial p(\mathbf{x}, t+\tau)}{\partial t}=D \nabla^{2} p(\mathbf{x}, t)+F[p(\mathbf{x}, t+\tau)]
$$

and this equation generalizes Eq. 3 .

We have introduced Eq. 5 in the simplest possible way (namely, by invoking the causality principle), but Eq. 5 can also be derived by analyzing the random motion of individuals with a characteristic mean rest time after birth (Fort and Méndez 1999a).

Assuming that the delay time $\tau$ is much smaller than the typical observation times, we expand Eq. 5 in powers of $\tau$. Discarding all terms in which $\tau$ appears to second or higher power gives the following:

$$
\begin{aligned}
\tau \frac{\partial^{2} p(\mathbf{x}, t)}{\partial t^{2}}+\frac{\partial p(\mathbf{x}, t)}{\partial t}= & D \nabla^{2} p(\mathbf{x}, t)+F[p(\mathbf{x}, t)] \\
& +\tau \frac{\partial F[p(\mathbf{x}, t)]}{\partial t} .
\end{aligned}
$$

Eq. 6 is called the hyperbolic reaction-diffusion equation (HRDE), and it is the center of the present paper. Note that it relies on keeping terms of Taylor expansions up to a higher order than within the classical (Fisher or PRDE) framework. For the full Eq. 5, Fort and Méndez (1999a) give an implicit formula for the speed of fronts for arbitrarily large $\tau$. Here, however, we will use Eq. 6 instead of Eq. 5. This makes it possible to find an explicit formula for $v$ which is more easy to use, and quite accurate in practice (Fort and Méndez 1999b).

One cannot use the HRDE to make any quantitative prediction unless one can estimate the value of the delay time $\tau$. A microscopic derivation of the HRDE (Eq. 6) (Fort and Méndez 1999a) has shown that, if $T$ is the mean time elapsed between two successive migrations (i.e., the generation time), then

$$
\tau=\frac{T}{2} .
$$

The microscopic theory then leads to a diffusion coefficient that is in agreement with previous work by many authors, namely,

$$
D=\frac{1}{4 T} \int_{-\infty}^{\infty} \int_{-\infty}^{\infty} \Phi(\Delta x, \Delta y) \Delta^{2} d \Delta x d \Delta y \equiv \frac{\overline{\Delta^{2}}}{4 T}
$$

where $\Delta^{2} \equiv \Delta x^{2}+\Delta y^{2}$ and $\Delta^{2}$ is the mean square displacement per jump (or migration). The function $\Phi(\Delta x$, $\Delta y)$ is the normalized probability distribution for a jump of length $\Delta$.

We use Eq. 6 not only because of conceptual arguments, but because it has been derived from a microscopic theory (Fort and Méndez 1999a). We stress that such an approach is necessary because it is precisely the microscopic derivation that makes it possible to identify the factor appearing in the first and last terms in Eq. 6 as half the mean time interval between subsequent migrations, $T$.

According to Eqs. 6 and 7, the density of individuals per unit area, $p(\mathbf{x}, t)$, evolves according to the HRDE:

$$
\begin{aligned}
\frac{T}{2} \frac{\partial^{2} p(\mathbf{x}, t)}{\partial t^{2}}+\frac{\partial p(\mathbf{x}, t)}{\partial t}= & D \nabla^{2} p(\mathbf{x}, t)+F[p(\mathbf{x}, t)] \\
& +\frac{T}{2} \frac{\partial F[p(\mathbf{x}, t)]}{\partial t}
\end{aligned}
$$

with the diffusion coefficient given by Eq. 8. Eq. 9 has the same form as the reaction-telegraph equation in Holmes (1993). However, in the case considered by Holmes (1993), the following expressions for the parameters $T$ and $D$ were used: 


$$
T_{\text {Holmes }}=\frac{1}{\lambda} \quad D_{\text {Holmes }}=\frac{\gamma^{2}}{2 \lambda} .
$$

Although the derivations in the present paper and in Holmes (1993) lead to the same equation (Eq. 9), they have been made in very different contexts because we do not assume a correlated random walk. Consequently, the meaning of the parameters $T$ and $D$ is rather different. Holmes's model assumes that individuals perform steps in one dimension whose lengths in space, $\gamma \lambda$, and in time, $\lambda$, are always the same ( $\gamma$ is the organism velocity), and then, the macroscopic delay terms in Eq. 9 come from the characteristic time to reverse direction, i.e., $1 / \lambda$. In contrast, in our model, which holds in two dimensions, the steps can have different lengths and the delay time is due to the assumption of a characteristic time interval $T$ during which newborn individuals do not disperse (Fort and Méndez 1999a).

In the limit in which the effect of the delay time is neglected $(T \rightarrow 0)$, Eq. 9 becomes Fisher's equation Eq. 3. It is well known that Fisher's equation allows instantaneous response and divergent flux. Time-delayed equations such as Eq. 9 were long ago introduced in the physics literature (Jou et al. 2001) to get around this problem. We must mention that this problem is also solved in the context of methods that rest on integrodifference equations by making use of dispersal kernels with finite support (Neubert and Caswell 2000). That approach in terms of multiple life stages, each with their own life traits and dispersal, has more generality than the approach we take in this paper. However, Eq. 9 has advantages over integrodifference equations in some situations. First, dispersal kernels can be applied only if the dispersion histogram of the species considered is known. In contrast, the diffusion coefficient (Eq. 8) can be estimated even if the dispersion histogram is not known (Lubina and Levin 1988), although it is true that its uncertainty increases in turn. Second, it is easier to estimate invasion speeds from Eq. 9, because a simple, explicit analytic expression for the speed can be applied.

Méndez and Camacho (1997) and Méndez, Fort and Farjas (1999) show that the asymptotic $(t \rightarrow \infty)$ speed of the wavefront solutions to Eq. 9 is given by

$$
v=\frac{2 \sqrt{D F^{\prime}(0)}}{1+\frac{T}{2} F^{\prime}(0)}
$$

where $F^{\prime}(0)=d F /\left.d p\right|_{p=0}$ is the intrinsic rate of population growth and conditions $v^{2}<2 D / T$ and $F^{\prime}(0) T / 2$ $<1$ must be satisfied (Méndez et al. 1999). In the limit $T \rightarrow 0$, Eq. 10 becomes Fisher's result, namely,

$$
v_{T \rightarrow 0}=2 \sqrt{F^{\prime}(0) D} .
$$

In the next sections, we compare the predictions of Eqs. 10 and 11 to observation of range expansions of Eurasian Collared-Doves and House Finches.

\section{COMPARISON TO OBSERVATIONS}

\section{House Finch}

In this section, we shall deal with the range expansion of the House Finch (Carpodacus mexicanus) in northern America, which has been taking place since 1940. First of all, we will compute the necessary parameters in order to make quantitative predictions. Then, we will apply Eqs. 10 and 11 to predict the speed of the invasion and compare their results to the corresponding observations.

Although it is not strictly necessary to do so, for the sake of clarity we assume that the source terms in Eqs. 9 and 3 make up a logistic function, namely,

$$
F(p)=a p\left(1-\frac{p}{p_{\max }}\right)
$$

where $a=d F /\left.d p\right|_{p=0}=F^{\prime}(0)$ is the intrinsic growth rate and $p_{\max }$ is the carrying capacity. Then, the front advance speed (Eq. 10) can be written as

$$
v=\frac{2 \sqrt{a D}}{1+\frac{a T}{2}}
$$

and Fisher's speed reads

$$
v=2 \sqrt{a D}
$$

We stress that Eq. 13 is the same result for the velocity as that appearing in Holmes (1993) provided that the expression above for $T_{\text {Holmes }}$ and $D_{\text {Holmes }}$ are used instead of our parameters $T$ and $D$, and that conditions $v^{2}<$ $2 D_{\text {Holmes }} / T_{\text {Holmes }}$ and $a T_{\text {Holmes }} / 2<1$ (mentioned below Eq. 10) are satisfied.

Bird reproductive dynamics.-The first parameter we must estimate is $a$. We note from Eq. 12 that for small population sizes $(P \approx 0)$, the global population size $P$ changes according to

$$
\frac{d P}{d t} \approx a P \text {. }
$$

Note that here we are dealing with the total population $P$, and not with the population density at a given locality $p(\mathbf{x}, t)$, so diffusive effects can be neglected. (The first term on the right-hand-side of Eqs. 9 and 3 corresponds to individuals incoming and/or outgoing from the system, and thus does not apply if we consider the whole continent, within which all individuals move.) This point is quite important in practice, because it means that in order to determine the value of $a$ one should make use of the whole-survey data and not only those of a restricted range. In the later case, the population change would be also affected by dispersal (first term in the right-hand side of Eqs. 9 and 3). Moreover, local climatology and geography can yield different reproductive dynamics. Thus, in contrast to previous work (Veit and Lewis 1996), we shall use whole-survey 


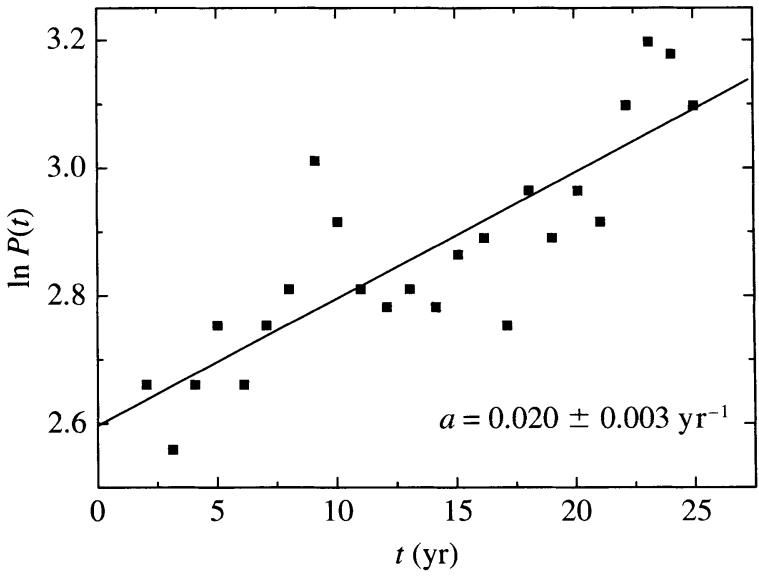

FIG. 1. House Finch survey-wide population numbers ( $P$, measured in counts per party hour) vs. time (USGS 2001). In order to estimate the intrinsic growth rate $a$ we used the logarithmic plot and removed points far away from the exponential phase (large times), which implied a decrease in the regression coefficient of the linear fits.

data below. We stress that this applies only to the initial, exponential stage of population growth.

Eq. 15 can easily be integrated to yield the following temporal evolution of the total population size:

$$
P(t)=P_{0} e^{a t}
$$

(if $P \rightarrow 0$ ) where $P_{0}=P(0)$.

From the observed population numbers (Fig. 1), we can estimate in a simple way the value of $a$, making use of Eq. 16. We have done so by fitting a straight line to the logarithms of the observed data (USGS, 2001) for the total population vs. time. The slope of the fitted line gives the value of $a$ (Fig. 1.), namely $a$ $=0.020 \pm 0.003 \mathrm{yr}^{-1}$ (mean $\pm 1 \mathrm{SE}$ ).

Estimation of the delay time.-The parameter $T$ appearing in Eqs. 9 and 13 is the mean time interval elapsed between two successive migrations (Fort and Méndez 1999a). Thus $T$ may be estimated as the time needed for a newborn individual to grow into an adult and reproduce. When the adult age is reached, individuals leave the paternal territory and fly to new places. The value of this time has been estimated from observations to lie between 1.5 and $2 \mathrm{yr}$ (Hochachka and Dhondt 2000), so we shall take as a typical value $1.75 \mathrm{yr}$. In fact, values of $T$ between 1.5 and $2 \mathrm{yr}$ do not change the results we will derive below.

Dispersal.-The diffusion coefficient can be estimated from Eq. 8. To determine the value of $\overline{\Delta^{2}}$ we use the histogram in Fig. 2. From these data, we can estimate the mean-squared displacement as

$$
\overline{\Delta^{2}}=\sum_{i} \Delta_{i}^{2} f_{i}
$$

where $\Delta_{i}$ are the observed distances and $f_{i}$ their respective observed frequencies $\left(\Sigma_{i} f_{i}=1\right)$. From Eq. 17 and Fig. 2, we get $\overline{\Delta^{2}}=(71 \pm 13) \times 10^{3} \mathrm{~km}^{2}$ and so

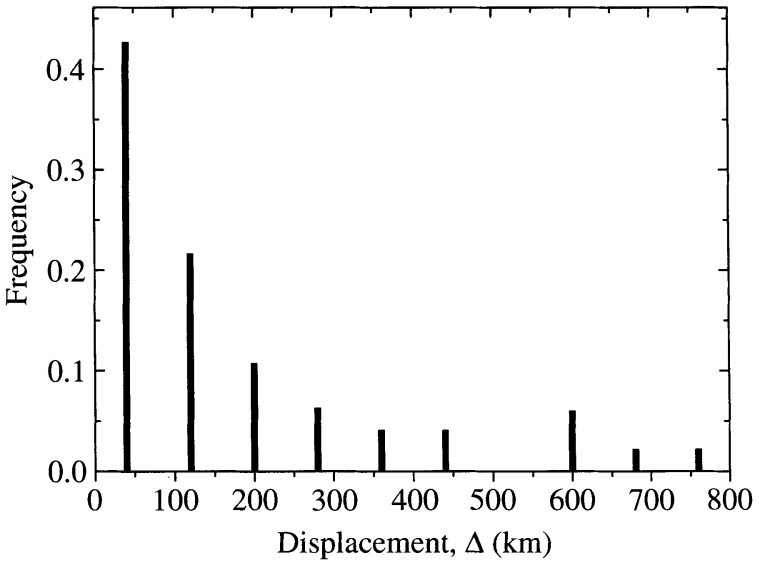

FIG. 2. House Finch annual dispersal histogram (from the data in Veit and Lewis [1996]). In agreement with our singlespecies model, we include adults as well as juveniles. Note that a time interval of one year, which is the value for the data shown, corresponds roughly to the time required by a newborn individual to turn into an adult (Hochachka and Dhondt 2000), i.e., roughly to parameter $T$ in our model

$\overline{\Delta^{2}} / T=(41 \pm 9) \times 10^{3} \mathrm{~km}^{2} / \mathrm{yr}$, which leads us to the diffusion coefficient:

$$
D=(10.1 \pm 2.4) \times 10^{3} \mathrm{~km}^{2} / \mathrm{yr} .
$$

Prediction of the front speed and comparison to the observed value.- Now that we have estimated the necessary parameter values, we can compute the front speed predicted by the time-delayed model, Eq. 9. This yields $v=28 \pm 4 \mathrm{~km} / \mathrm{yr}$. The observed speed, $v_{\exp }=$ $28 \pm 1 \mathrm{~km} / \mathrm{yr}$ (Okubo 1986), agrees rather well with the value predicted above (Fig. 3). Fig. 4 shows the values of the parameters $a$ and $\overline{\Delta^{2}} / T$ that are compatible with the observed speed. Many combinations of these

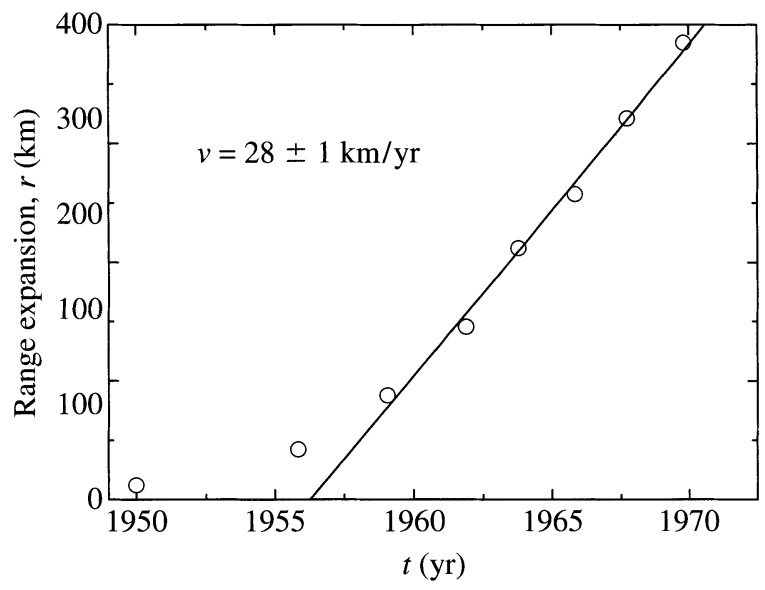

FIG. 3. Linear fit of experimental data (Okubo 1986) to estimate the speed of the range expansion of the House Finch, $v_{\text {exp }}$. The first two data points have been removed because they correspond to early times, when the initial population has not reached the saturation value, and therefore the front speed is not yet the asymptotic one. 


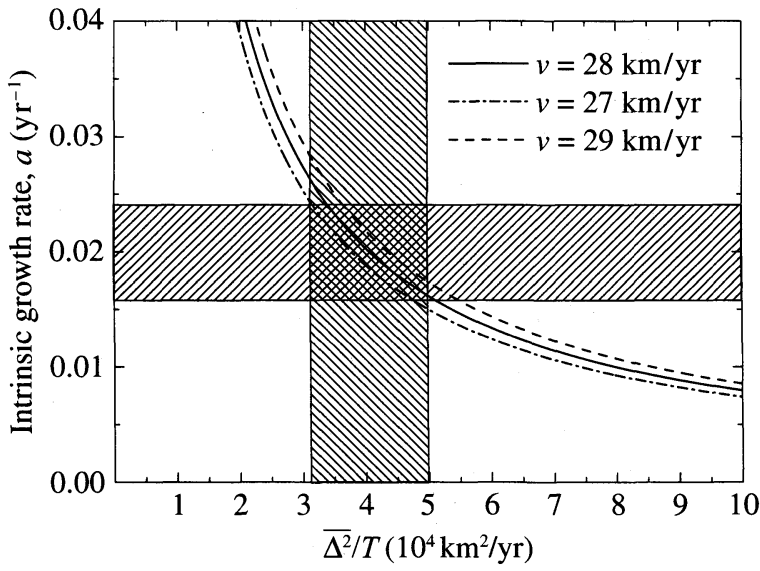

FIG. 4. Results from applying the time-delayed model (Eq. 13) to the House Finch expansion. The shaded regions correspond to the possible ranges of the parameter values, according to independent observations. The outer curves limit the predicted values for the front speed. It is seen that the theory predicts, for many of the possible values of the parameters, a speed that is within the observed range $(28 \pm 1$ $\mathrm{km} / \mathrm{yr}$ ). No free or adjustable parameters have been used.

parameters, obtained from the observations in Figs. 1 and 2 (shaded areas in Fig. 4), yield a range expansion speed consistent with that observed (Fig. 3).

\section{Eurasian Collared-Dove}

We now turn our attention to the range expansion of Eurasian Collared-Dove (Streptopelia decaocto Friv.) into Europe. Using the same method as for the House Finch, the graph of survey-wide data for the dove (USGS 2001) allows us compute $a=0.29 \pm 0.02 \mathrm{yr}^{-1}$ (see Fig. 5). Van Den Bosch et al. (1992: Tables 3 and 4) report: $T=1.81 \pm 0.73 \mathrm{yr}$ and $\overline{\Delta^{2}}=(36 \pm 10) \times$ $10^{3} \mathrm{~km}^{2}$. Thus, $D=5026 \pm 2400 \mathrm{~km}^{2} / \mathrm{yr}$, and the speed (Eq. 13) predicted by HRDE is $v=60 \pm 19 \mathrm{~km}^{2} / \mathrm{yr}$, whereas the classical speed (Eq. 14) is $v_{T \rightarrow 0}=76 \pm 19$ $\mathrm{km}^{2} / \mathrm{yr}$. Van Den Bosch et al. (1992) also report that the observed velocity of the Eurasian Collared-Dove population expansion is $v_{\exp }=44 \pm 3 \mathrm{~km}^{2} / \mathrm{yr}$. Note that the time-delayed model gives a range for the front speed compatible with observations, whereas Fisher's model does not. Fig. 6 shows the values of the parameters $a$ and $\overline{\Delta^{2}} / T$ that are compatible with the observed speed for the Eurasian Collared-Dove expansion.

\section{DISCUSSION}

In this paper we have shown that the House Finch and Eurasian Collared-Dove invasion speeds can be explained (Figs. 4 and 6) by a simple time-delayed model. Our model provides an analytical result (Eq. 10), which reduces to Fisher's classical value (Eq. 11) in the appropriate limit. It is interesting to point out that several other models have been put forward in connection with the House Finch invasion. Veit and Lewis (1996) made use of a discrete-time model with

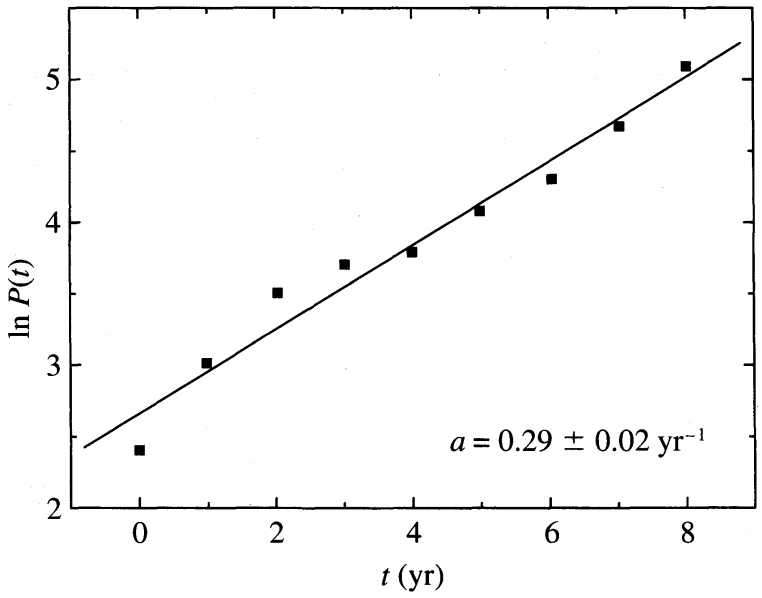

FIG. 5. Eurasian Collared-Dove survey-wide population numbers $(P)$ vs. time $t$ (USGS 2001). In order to estimate the intrinsic growth rate $a$ we used the logarithmic plot and removed points far away from the exponential phase (large times) that implied a decrease in the regression coefficient of the linear fits.

two stages (adults and juveniles) and, in contrast to Eq. 12 , they did not consider a logistic growth term. Their focus was fundamentally different from ours: their purpose was to explain the initial acceleration of the invasion, so they do not derive a simple formula for the speed over long periods. Shigesada and Kawasaki (1997) took into account the habitat preference of the House Finch in some regions. Theirs is also a very interesting, more detailed approach, which uses the observed range expansion speed as an input parameter. In contrast, we have predicted its value from theory and seen that it is close to that observed.

We now turn to the relevance of the time-delayed model (Eqs. 9 and 10) as compared to Fisher's model (Eqs. 3 and 11). First we notice that for the House Finch expansion analyzed in the previous section, the

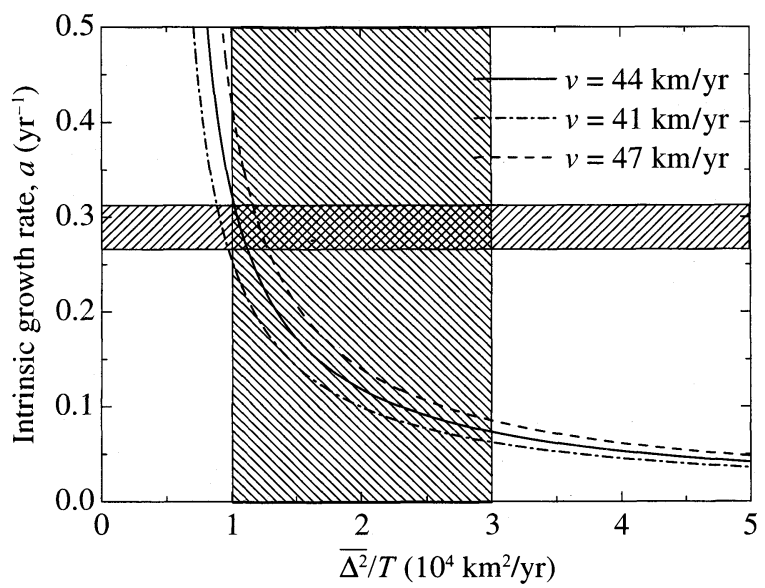

FIG. 6. Results from applying the time-delayed model (Eq. 13) to the Eurasian Collared-Dove expansion. See Fig. 4 for explanation of shaded areas and curves. 
TABLE 1. Comparison between the time-delayed and Fisher's models for range expansions of several biological species.

\begin{tabular}{lccc}
\hline \hline \multicolumn{1}{c}{ Parameter } & $\begin{array}{c}\text { Preindustrial } \\
\text { farmers (Fort and } \\
\text { Méndez 1999a) }\end{array}$ & $\begin{array}{c}\text { House Finch } \\
\text { (Carpodacus } \\
\text { mexicanus) }\end{array}$ & $\begin{array}{c}\text { Eurasian Collared-Dove } \\
\text { (Streptopelia } \\
\text { decaocto Friv.) }\end{array}$ \\
\hline$T(\mathrm{yr})$ & 25 & $1.75 \pm 0.25$ & $1.81 \pm 0.73$ \\
$D\left(\mathrm{~km}^{2} / \mathrm{yr}\right)$ & 15.44 & $10100 \pm 2400$ & $5026 \pm 2400$ \\
$a\left(\mathrm{yr}^{-1}\right)$ & 0.032 & $0.020 \pm 0.003$ & $0.29 \pm 0.02$ \\
$v_{T \rightarrow 0}(\mathrm{~km} / \mathrm{yr})$ & 1.41 & $28 \pm 4$ & $76 \pm 19$ \\
$v(\mathrm{~km} / \mathrm{yr})$ & 1.00 & $28 \pm 4$ & $60 \pm 19$ \\
$\left(v_{T \rightarrow 0}-v\right) /(v)$ & & $1.8 \pm 0.4$ & $26 \pm 11$ \\
$a T / 2(\%)$ & 41 & $28 \pm 1$ & $44 \pm 3$ \\
$v_{\text {exp }}(\mathrm{km} / \mathrm{yr})$ & $1.0 \pm 0.2$ & & \\
\hline
\end{tabular}

Note: All values stated with an error term are mean $\pm 1 \mathrm{SE}$.

speed predicted in the framework of the classical theory due to Fisher (Eq. 11) is different from the time-delayed prediction (Eq. 10, used above) by only $\sim 2 \%$. This is due to the small value of the product of the delay time $T$ and the initial growth rate $a$, which is much lower than unity in this specific example. In fact, if we compare Eq. 10 to Eq. 11 , it is easy to show that $\left(v_{T \rightarrow 0}-\right.$ $v) / v=\left(F^{\prime}(0) T\right) / 2$. Therefore, for this particular species, Fisher's model provides a relatively adequate framework (provided that the parameter values are carefully evaluated, as done in the present paper). However, the validity of Fisher's approximation breaks down for other species. In some cases, such as human populations, $T$ is much higher and the correction of the time-delayed model, as compared to Fisher's, is $\sim 40 \%$ (Table 1: column 1). In other cases, including the Eurasian Collared-Dove, $a$ can be much larger. As shown in the last column in Table 1, this happens for the dove: in contrast to Fisher's model, our time-delayed theory then provides a range for the speed that is consistent with the experimental value. To the best of our knowledge, this is the first time that such an important correction has been derived for a nonhuman species. Therefore, in order to progress toward the unification of the theory of range expansions, it is in general necessary to take into account the role of the delay time. We stress that Holmes (1993) finds negligible corrections for all species she studied simply because the effect she analyzed (namely, correlations in direction of motion between subsequent steps) is completely different from ours (i.e., a rest time between subsequent migrations). This is why the value of $T$ for the House Finch in Holmes (1993) is different from ours. The value of $a$ is also different because Holmes (1993) used life table data instead of whole-survey numbers (Fig. 1 in the present paper). Besides, the value of $D$ in Holmes (1993) is different, because it is based on an equation in Holmes (1993: Eq. 19), which in fact holds in diffusion, not in reaction-diffusion systems, and for a very specific initial condition (see its derivation in Shigesada and Kawasaki 1997, Section 3.2).

Here, we have presented an example of each of the three distinct possible situations (Table 1): (1) in some cases, $a T / 2$ is much smaller than unity and Fisher's model is a fair approximation (e.g., when dealing with the House Finch expansion). In other cases, this is not so, either because (2) $a$ is much larger (e.g., in the Eurasian Collared-Dove expansion), or (3) because $T$ is larger (e.g., in human population expansions). Thus, whenever the value of the product $a \times T$ is high enough (say, $a \times T>0.2$ ), the delay effect should be taken into account since it will induce an important change to Fisher's prediction. Therefore, it seems essential to take Eq. 9 into account in future studies of biological range expansions.

\section{ACKNOWLEDGMENTS}

We gratefully acknowledge Prof. Michael Neubert for helpful suggestions for improving this manuscript and Prof. David Jou for discussions. This work has been partially funded by the Ministry of Science and Technology under grants BFM 2000-0351-C03-01 (all authors), SGR 2001-00186 (all authors) and REN 2000-1621 CLI (J. Fort).

\section{Literature Cited}

Ammerman, A., and L. L. Cavalli-Sforza. 1984. The Neolithic transition and the genetics of populations in Europe. Princeton University Press, Princeton, New Jersey, USA.

Fisher, R. A. 1937. The wave of advance of advantageous genes. Annals of Eugenics (London) 7:355-369.

Fort, J. 2003. Wave of advance model of the Austronesian population expansion. Antiquity, in press.

Fort, J., and V. Méndez. 1999a. Time-delayed theory of the Neolithic transition in Europe. Physical Review Letters 82: 867.

Fort, I., and V. Méndez. 1999b. Reaction-diffusion waves of advance in the transition to agricultural economics. Physical Review E 60:5894-5901.

Fort, J., T. Pujol, and L. L. Cavalli-Sforza, 2003. Paleolithic expansions waves of advance. Cambridge Archaeological Journal 13, in press.

Hengeveld, R. 1989. Dynamics of biological invasions. Chapman and Hall, London, UK.

$\rightarrow$ Hochachka, W., and A. Dhondt, 2000. Density-dependent decline of host abundance resulting from a new infectious disease. Publication of the National Academy of Sciences (USA) 97:5303-5306.

Holmes, E. E. 1993. Are diffusion models too simple? A comparison with telegraph models of invasion. American Naturalist 142:779-795.

Jou, D., J. Casas-Vázquez, and G. Lebon. 2001. Extended irreversible thermodynamics. Third edition. Springer, Berlin, Germany. 
Kot, M. 1992. Discrete-time travelling waves: ecological examples. Journal of Mathematical Biology 30:413-43.

Kot, M., M. A. Lewis, and P. van den Driessche. 1996. Dispersal data and the spread of invading organisms. Ecology 77:2027-2042.

Lubina, J., and S. A. Levin. 1988. The spread rate of reinvading organisms: range expansion of the California sea otter. American Naturalist 131:526-543.

Méndez, V., and J. Camacho. 1997. Dynamics and thermodynamics of delayed population growth. Physical Review E 55:6476-6482.

Méndez, V., J. Fort, and J. Farjas. 1999. Speed of wave-front solutions to hyperbolic reaction-diffusion equations. Physical Review E 60:5231-5243.

Neubert, M., and H. Caswell. 2000. Demography and dispersal: calculation and sensitivity analysis of invasion speed for structured populations. Ecology 81:1613-1628.

Okubo, A. 1986. Diffusion-type models for avian range expansion. International Ornithological Congress 19(1): 1038-1049.
Rendine, S., A. Piazza, and L. L. Cavalli-Sforza. 1986. Simulation and separation by principal components of multiple demic expansions in Europe. American Naturalist 128:681705(1986).

Shigesada, N., and K. Kawasaki. 1997. Biological invasions: theory and practice. Oxford University Press, New York, New York, USA.

USGS. 2001. The North American Breeding Bird Survey. Graph of survey-wide yearly indices from CBC. USGS Patuxent Wildlife Research Center, Laurel, Maryland, USA.

Van Den Bosch, F., R. Hengeveld, and J. A. J. Metz. 1992. Analysing the velocity of animal range expansion. Journal of Biogeography 19:135-150.

Van Den Bosch, F., J. A. J. Metz, and O. Diekmann. 1990. The velocity of spatial population expansion. Journal of Mathematical Biology. 28:529-565.

Veit, R. R., and M. A. Lewis. 1996. Dispersal, population growth and the Allee effect: dynamics of the house finch invasion of Eastern North America. American Naturalist 48:255-274. 